\title{
An Optimized Clustering Algorithm for Contour Data
}

\author{
Yucheng CHU and Lizhen WANG ${ }^{1}$ \\ School of Information Science and Engineering, Yunnan University, Kunming 650500, \\ China
}

\begin{abstract}
The initial center's selection of the traditional K-means algorithm is random. It makes the algorithm instability. Traditional clustering methods are hard to apply to the clustering of contour data. The data from the contour data set forms the contour shape, but the cluster center is not on the contour. The final cluster center is in the inner center of the contour. Using K-means algorithm directly is not able to handle this kind of data well. In order to optimize the initial clustering center of the contour data, and in order to solve the problem of contour data clustering. Based on the high density area may be initial clustering centers. And under the thought of the farther apart distance the more likely belonged to different clustering. In this paper, an algorithm of contour data clustering is proposed. This algorithm can obtain better initial central for contour data, so as to improve the clustering effect of contour data. Firstly, the algorithm calculates the distance between the samples. And based on the premise of the two farthest points most probability does not belong to the same class. The algorithm finds out the farthest two points according to the sample distance. With one point as the center, find the point closest to the point to join the point set. Until the data set number greater than or equal to $\alpha$ number ( $\alpha$ is the ratio of the number of samples to the number of clusters). Calculate the average of all points as the initial cluster center. Repeat the above steps to get $\mathrm{K}$ initial cluster centers. Then the final clustering calculation is carried out according to the K-means algorithm. After the experiment on the experimental data set, the improved algorithm has a strong stability. The initial clustering center is uniformly discrete. And the clustering results have a high accuracy and have a good F1 value. The improved algorithm solves the clustering problem of contour data well.
\end{abstract}

Keywords. Contour data, Initial cluster center, K-means clustering algorithm.

\section{Introduction}

Clustering is a kind of unsupervised learning method, which divides data into different data classes according to different data characteristics. Individuals belonging to different data classes have different data characteristics, while individuals belonging to the same data class have same or similar data characteristics. The purpose of clustering is to divide same or similar data individuals into a cluster and assign different categories of individuals to different clusters [1-5]. Traditional clustering algorithms can be divided into partition-based clustering [6], density-based clustering [7], hierarchy-based clustering [8], grid-based clustering [9], etc. K-means algorithm is a

\footnotetext{
${ }^{1}$ Corresponding Author: Lizhen Wang, School of Information Science and Engineering, Yunnan University, Kunming 650500, China; E-mail: lzhwang2005@126.com.
} 
classically clustering algorithm based on partition. As an indirect clustering method based on the similarity measure between samples, k-means algorithm takes the number of cluster classes as a parameter and divides $n$ data objects in the data set into $\mathrm{K}$ clusters according to rules, so that the similarity within the cluster is higher while the similarity between clusters is lower. Although K-means algorithm has the advantages of simple, efficient and easy to understand, it still has many shortcomings. It can only deal with spherical or globular data sets, unstable initial clustering center [10], easy to fall into local optimal solutions, and so on. Aiming at the shortcomings of this algorithm, many scholars have made many improvements. In literature [11], in order to solve the sensitivity problem of the initial clustering center, layered agglomeration clustering algorithm [12] was adopted to select the initial clustering center to ensure the high quality of the central point. Literature [13] proposed an improved initial clustering center selection algorithm for K-means algorithm, which introduced the idea of high-density priority clustering [14], for the sensitivity of initial clustering center and the inability to handle data sets with large density differences. Contour data is a kind of classic data type. Its data form the contour shape, beautiful contour data but not too convenient to handle. But the final clustering center of this kind of data is not on the outline of the data. The final cluster center is usually located on the inner center of the contour. So it's not always good to have a data point as the initial cluster center. Cause the final cluster center is also usually located in the center of the contour. Therefore, the optimization of the initial cluster center is particularly important. Experiments show that the improved algorithm has good ability to deal with contour shape data. The improved algorithm in this paper has strong anti-interference ability due to the operation mechanism. The clustering result is more stable. Compared to the traditional K-means, K-means++, it has good stability and good profile coefficient. This paper fully demonstrated the improved algorithm is feasible, reasonable and effective.

Due to the K-means algorithm is sensitive to the initial clustering center. It can't handle the contour data directly [15]. In order to solve the initial cluster center selection problem of contour data, to improve the feasibility of clustering, enhanced clustering effect, etc. This paper proposes a new simple and effective optimization algorithm. This algorithm can greatly reduce the random selection of the impact of the initial clustering center. We can use the algorithm to select the better initial clustering center. It is very suitable for contour shape clustering analysis of data to find out the initial clustering center which are located in the center of the contour. The algorithm combines the thought that the high density area at the same time may have the clustering center and the thought that the farther away the points are, the more likely they are to belong to different clusters. The algorithm is simple and quick, only need to deal with the distance of all points for the first time. It can speed up the convergence rate of the clustering, enhance the stability of the algorithm, discrete the initial clustering center of the uniform, improves the clustering effect.

\section{The basic idea of the improved algorithm}

Before describing the basic idea of this algorithm, first we need to know about the basic idea of K-means algorithm [16]. The algorithm proposed in this paper needs to use K-means algorithm. K-means algorithm idea is as follows: first of all, $\mathrm{K}$ data objects are selected randomly on the data set as the initial clustering center. And then calculate the Euclidean distance of each data object with the center and division to 
the center of the smallest distance. Form $\mathrm{K}$ clusters and recalculate the new cluster center. Repeat the above steps until the clustering center are no longer changes or the error sum of squares of the difference of two adjacent clustering results is less than a threshold value.

This article combines the thought that high density regions may have clustering center and the thought that the farther apart the points are, the less likely they are to belong to the same cluster. Firstly, calculate the distance between samples. According to the sample distance form collection, find out the nearest two points. Then according to the calculation formula of collection point to find out all the other point closest to the initial point. Find all relevant data points until the set number greater than or equal toanumber ( $\alpha$ is the ratio of the number of samples to the number of clusters). Calculate the mean of all the collection points to get the first initial cluster center. Repeat the above steps to get $\mathrm{K}$ ( $\mathrm{K}$ is clustering number) collections. Calculate the mean of each set as the initial center. And the final clustering result is obtained by the K-means algorithm. Therefore, the improved algorithm inherits the characteristic that the farthest point is least likely to belong to the same class. And it integrates the characteristic of high-density priority clustering. It discretizes the initial clustering center of contour data uniformly. It increases the ability of the algorithm to resist the interference of outliers. And it can obtain the clustering center that is more consistent with the final clustering result. It can be used to process contour data.

Let's say I have $\mathrm{n}$ sample data sets: $D=\left\{x_{1}, x_{2}, \ldots, x_{n}\right\}$, There are $\mathrm{K}$ clusters, $C=$ $\left\{C_{1}, C_{2}, \ldots, C_{k}\right\}, m$ collections: $M=\left\{M_{1}, M_{2}, \ldots, M_{m}\right\}$.

Definition 1: The Euclidian distance between two data objects is.

$$
d\left(x_{i}, x_{j}\right)=\sqrt{\sum_{l=1}^{n}\left(x_{i l}-x_{j l}\right)^{2}}
$$

Where, $x_{i}$ and $x_{j}$ are data objects, $x_{i l}$ is the $l$ attributes of $x_{i}, x_{j l}$ is the $l$ attributes of $x_{j}$.

Definition 2: The cluster center (Centerk) of the $k$ th cluster is defined as.

$$
\text { centerk }=\frac{1}{\left|C_{k}\right|} \sum_{\mathrm{i}=1}^{\left|C_{k}\right|} x_{i}, x_{i} \in C_{k}
$$

Where, Centerk represents the center of the $k$ th cluster, $C_{k}$ represents the $k$ th cluster, and $x_{i} \in C_{k}$ represents the data object $x_{i}$ belongs to $C_{k} .\left|C_{k}\right|$ represents the number of points in $C_{k}$.

Definition 3: The objective function is the sum of squares of errors

$$
E=\sum_{j=1}^{k} \sum_{i=1}^{\left|C_{k}\right|} d\left(x_{i}, \text { Centerk }\right), x_{i} \in C_{k}
$$

In K-means algorithm, the similarity between data objects is calculated by Euclid distance. The smaller the distance, the higher the similarity between the data objects. On the uneven density data set, the higher the density the more likely it is to 
get together. In order to combine the density and distance, find $\mathrm{K}$ discrete points respectively. We go through the furthest point to ensure that the clustering centers are discrete. And we go through high density center point set to calculate the mean value. It is not only benefit the objective function of convergence, and is advantageous to the clustering of the accurate and reliable.

According to the above principles, the steps to find discrete and uniformly distributed $\mathrm{K}$ points as the initial clustering center on the data space distribution are as follows.

(1) Calculate the Euclidean distance between two data objects $d\left(x_{i}, x_{j}\right), i, j=1$, $2, \ldots, n$, find the two most distant data objects $d 1$ and $d 2$ and delete them from the total data set $D$.

(2) Find $\alpha(n / k)$ points closest to $d 1$ to form a set $n d 1$ and remove them from the total dataset D. According to formula (2), the initial cluster center is calculated and added to the $K$ set of the initial cluster center.

(3) Find $\alpha(n / k)$ points closest to $d 2$ to form a set $n d 2$ and remove them from the total dataset D. According to formula (2), the initial cluster center is calculated and added to the $K$ set of the initial cluster center.

(4) Repeat (1) (3) until the number of $K$ sets is greater than or equal to $K-1$.

(5) When the number of initial center points in the $K$ set is equal to $K$-1, then formula (2) is used to calculate the initial clustering center points of the remaining points and add them to the initial clustering center $K$ set. When the number of initial center points in $K$ set is equal to $K$, then the process of searching for initial center points of clustering ends.

\section{Examples and applications of the improved algorithm}

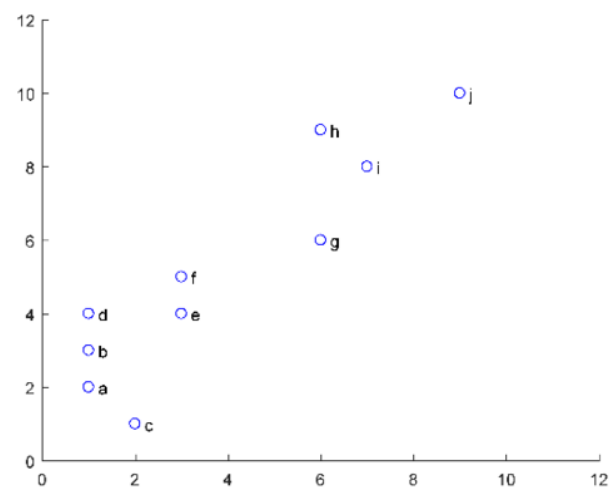

Figure 1. A data distribution diagram.

For example, this is a 2-dimensional dataset $W$ with a data size of 10 , and its data distribution is shown in Figure 1.

Now if we need to divide the data set into three categories, we will find the initial clustering center according to the idea of this algorithm. So first of all find out the most remote point, the figure 1 shows that a and $\mathrm{j}$ furthest distance, and then respectively from a recent find three data points, the distance is obtained by Formula 
(1) a's recent three data points $\mathrm{b}, \mathrm{c}, \mathrm{d}$, adding them and point a to $N_{\varphi d l}$ collection, calculated by Formula (2) as the initial clustering center of K-means clustering center, one of the K concentration and add to the initial clustering center. Then find the three data points closest to $\mathrm{J}$, and get the three data points closest to $\mathrm{J}$ through Formula (1), add them and point j to $N_{\varphi d 2}$ set. Calculate the clustering center through Formula (2) as one of the initial clustering centers of K-means, and add it to the initial clustering center $K$ set. Since the number of initial center points in the $K$ set is equal to $K-1$, then the initial clustering center points of the remaining points are calculated by Formula (2), and added to the initial clustering center $K$ set, and the process of finding the initial clustering center point is completed.

But how can it be used in the contour data. For example, there is a 2-dimensional circular data set $G$ with a data size of 16 , and its data distribution is shown in Figure 2. The data constitutes two rings on the left and the right. We can predict that the final clustering center should be the center of the circle.

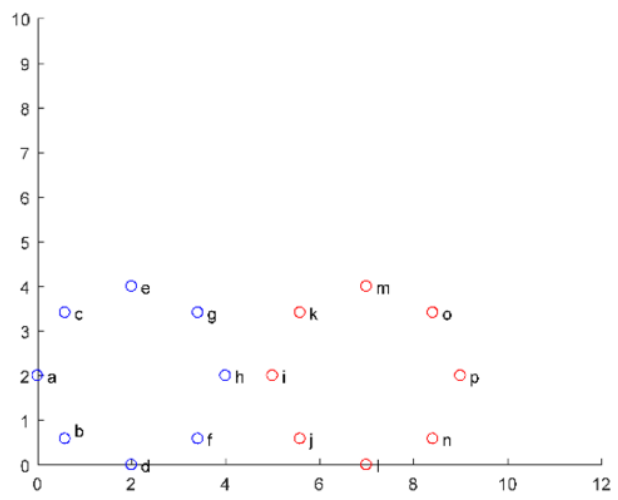

Figure 2. Data distribution diagram2.

Suppose that they need to be divided into two categories, then if the traditional $\mathrm{K}$-means clustering algorithm is adopted, the algorithm has great randomness, and it is difficult to get a good initial clustering center, and the clustering problem of such contour data will not be handled well in the end. According to the improved algorithm proposed in this paper, the initial clustering center is found. It can be seen from Figure 2 that points $\mathrm{A}$ and $\mathrm{P}$ are the farthest from each other, so the seven data points closest to A are found respectively. According to Formula (1), the data points closest to A are B, C, D, E, F, G and $\mathrm{H}$. Adding them and point a to $N_{\varphi d l}$ collection. The clustering center is calculated by formula (2) as one of the initial clustering centers of K-means, and is added to the initial clustering center $K$ set. Then, find the 7 data points closest to $\mathrm{P}$, and get the 7 data points closest to $\mathrm{P}$ through Formula (1): I, J, $\mathrm{K}, \mathrm{L}, \mathrm{M}, \mathrm{N}, \mathrm{O}$. Adding them and point a to $N_{\varphi d 2}$ collection. The clustering center is calculated by Formula (2) as one of the initial clustering centers of K-means, and is added to the initial clustering center $K$ set. Therefore, since the number of initial center points in the current $K$ set is equal to $K$, the process of searching for initial cluster center point's ends. The obtained initial clustering center points are about the center positions of the two rings, so it is easy to carry out subsequent K-means clustering, and the final clustering result is that the left and right two rings belong to two classes, and the clustering effect is very good. 
For another example, the data in Figure 2 can be replaced with other contour data, such as square, triangle, polygon, etc. And the size of contour band can be changed, which can be attempted by this algorithm. If the distribution of each contour data volume is uniform, the algorithm will get a good clustering effect.

\section{The improved algorithm is described in detail}

If the data set $N=\left\{x_{1}, x_{2}, \ldots, x_{n}\right\}$, contains $n$ data objects, each data object has $S$ dimension. The detailed description of the improved algorithm is as follows (the parameter $\varphi$ is the ratio of the total number of data set objects to the number of clustering centers, threshold $\beta$ refers to the difference of the sum of the squares of the two adjacent errors) .

Input: Data set $N=\left\{x_{1}, x_{2}, \ldots, x_{n}\right\}$, Number of cluster classes $K$, threshold $\beta$.

Output: Clustering results.

Methods:

(1) According to the method proposed in this paper to find initial cluster centers, $K$ cluster centers are selected as the initial centers.

(2) Calculate the distance between each data object and the center according to Formula (1), divide it into the nearest cluster center, and get $\mathrm{K}$ cluster classes.

(3) Recalculate the center of each cluster class according to Formula (2).

(4) Reclassify the clusters and update the centers.

(5) Until the cluster centers no longer change or the difference value of $E$ value for two consecutive times is less than the threshold value $\beta$.

(6) End of algorithm.

\section{Experimental results and analysis}

\subsection{The experimental data}

In order to verify the feasibility and effectiveness of the improved algorithm proposed in this paper, the experiment selected the Wine in the UCI database, Hayes Roth, Iris, airport, Ionosphere, Hagerman data set for the experiment and analysis. The dimensions of data sets are from several dimensions to a dozen dimensions. And the size of the data set range from hundreds to thousands of, which reflects the effect of the improved algorithm to cluster the data in different dimensions and size. It makes the results more convincing. The data set details are shown in Table 1. 
Table 1. Basic information of the dataset.

\begin{tabular}{cccc}
\hline The data set name & Data set size & Data dimension & Data categories \\
\hline Wine & 178 & 13 & 3 \\
Hayes-Roth & 132 & 5 & 3 \\
Iris & 150 & 4 & 3 \\
Tae & 151 & 5 & 3 \\
Ionosphere & 351 & 34 & 2 \\
Hagerman & 306 & 3 & 2 \\
\hline
\end{tabular}

\subsection{The experimental results}

In order to verify the effectiveness of the improved algorithm, the traditional K-means algorithm and K-Means ++ algorithm are used to compare with the improved algorithm proposed in this paper. In the experiment, precision, recall, F1 and contour coefficient were used to evaluate the clustering results of the algorithm. Accuracy rate refers to the proportion of the correct clustering to be positive in all the clustering results. Its value is generally in the interval of $[0,1]$. The larger the value means the more data are correctly clustered. The recall rate refers to the ratio between the correctly clustered positive and all positive samples, which is generally in the interval of $[0,1]$. F1 value is the harmonic mean of precision rate and recall rate. $\mathrm{F} 1$ value is generally in the interval of $[0,1]$. The $\mathrm{F} 1$ closer to 1 , the better the clustering. Contour coefficient is a way to evaluate the clustering effect. The value of SC is in the interval $[-1,1]$. The larger the value is, the better the clustering effect is. The calculation formula of precision rate, recall rate and $\mathrm{F} 1$ value is as follows.

$$
P=\frac{T p}{T p+F p} ; \quad R=\frac{T p}{T p+F \mathrm{n}} \quad ; \quad F 1=\frac{2 P R}{P+R}
$$

Where, $\mathrm{P}$ is the accuracy rate, $\mathrm{R}$ is the recall rate. $\mathrm{Tp}$ : the sample is positive and the clustering result is positive. Fp: The sample was negative and the clustering result was positive. Tn: The sample is negative and the clustering result is negative. Fn: The sample is positive and the clustering result is negative.

The contour coefficient (SC) of the data object can be obtained by the following formula.

$$
S(i)=\frac{b(i)-a(i)}{\max \{a(i), b(i)\}},(i=1,2, \ldots, n)
$$

Where, $a(i)$ represents the average distance between the number $\mathrm{i}$ data object and other points in the cluster to which it belongs, $b(i)$ represents the minimum value of the average distance between the number i data object and all points that are not in its own cluster, and $S(i)$ represents the contour coefficient of any data object. Finally, the contour coefficient $S C$ we need is obtained by averaging the entire $S(i)$.

In order to verify the clustering effect of the improved algorithm, six commonly used data sets of UCI are adopted as experimental data, and the experimental results of the improved algorithm are compared with the traditional K-means algorithm and K-means ++ algorithm, as shown in Table 2 Table 4. 
Table 2. Comparison of accuracy and stability of data results.

\begin{tabular}{|c|c|c|c|c|c|c|c|}
\hline \multirow[t]{2}{*}{ Algorithm } & & \multicolumn{2}{|c|}{ Wine } & \multicolumn{2}{|c|}{ Hayes-Roth } & \multicolumn{2}{|c|}{ Iris } \\
\hline & & $\begin{array}{c}\text { The initial } \\
\text { center }\end{array}$ & accuracy & $\begin{array}{c}\text { The initial } \\
\text { center }\end{array}$ & accuracy & $\begin{array}{c}\text { The initial } \\
\text { center }\end{array}$ & accuracy \\
\hline \multirow[t]{6}{*}{ K-means } & 1 & $(74,86,80)$ & 0.478 & $(122,69,117)$ & 0.273 & $(69,44,78)$ & 0.240 \\
\hline & 2 & $(118,17,103)$ & 0.169 & $(6,72,95)$ & 0.386 & $(18,146,5)$ & 0.473 \\
\hline & 3 & $(42,80,8)$ & 0.702 & $(29,51,105)$ & 0.280 & $(107,120,83)$ & 0.320 \\
\hline & 4 & $(139,141,9)$ & 0.112 & $(115,77,80)$ & 0.424 & $(26,146,22)$ & 0.500 \\
\hline & 5 & $(175,97,47)$ & 0.354 & $(130,115,94)$ & 0.295 & $(30,100,145)$ & 0.440 \\
\hline & Ave & - & 0.363 & - & 0.332 & - & 0.395 \\
\hline \multirow{6}{*}{$\begin{array}{c}\text { K-means } \\
++\end{array}$} & 1 & $(127,17,89)$ & 0.112 & $(77,131,66)$ & 0.280 & $(144,149,16)$ & 0.320 \\
\hline & 2 & $(176,177,16)$ & 0.500 & $(96,131,21)$ & 0.333 & $(39,149,60)$ & 0.447 \\
\hline & 3 & $(27,177,103)$ & 0.478 & $(122,131,48)$ & 0.394 & $(92,149,68)$ & 0.440 \\
\hline & 4 & $(98,177,16)$ & 0.354 & $(88,131,69)$ & 0.379 & $(106,149,11)$ & 0.02 \\
\hline & 5 & $(3,177,82)$ & 0.702 & $(120,131,7)$ & 0.280 & $(4,149,133)$ & 0.887 \\
\hline & Ave & - & 0.429 & - & 0.333 & - & 0.423 \\
\hline $\begin{array}{l}\text { Improved } \\
\text { algorithm }\end{array}$ & 1 & - & 0.702 & - & 0.439 & - & 0.887 \\
\hline \multirow[t]{2}{*}{ Algorithm } & & \multicolumn{2}{|c|}{ Tae } & \multicolumn{2}{|c|}{ Ionosphere } & \multicolumn{2}{|c|}{ Hagerman } \\
\hline & & $\begin{array}{c}\text { The initial } \\
\text { center }\end{array}$ & accuracy & $\begin{array}{c}\text { The initial } \\
\text { center }\end{array}$ & accuracy & $\begin{array}{c}\text { The initial } \\
\text { center }\end{array}$ & accuracy \\
\hline \multirow[t]{6}{*}{ K-means } & 1 & $(102,44,125)$ & 0.318 & $(269,184)$ & 0.644 & $(39,120)$ & 0.500 \\
\hline & 2 & $(94,91,102)$ & 0.371 & $(289,216)$ & 0.291 & $(18,296)$ & 0.516 \\
\hline & 3 & $(116,29,2)$ & 0.298 & $(10,239)$ & 0.709 & $(177,113)$ & 0.242 \\
\hline & 4 & $(48,69,58)$ & 0.358 & $(44,46)$ & 0.288 & $(127,43)$ & 0.500 \\
\hline & 5 & $(49,89,116)$ & 0.338 & $(250,264)$ & 0.712 & $(10,100)$ & 0.480 \\
\hline & Ave & - & 0.337 & - & 0.529 & - & 0.448 \\
\hline \multirow{6}{*}{$\begin{array}{c}\text { K-means } \\
++\end{array}$} & 1 & $(3,150,92)$ & 0.351 & $(69,350)$ & 0.709 & $(39,305)$ & 0.520 \\
\hline & 2 & $(61,150,141)$ & 0.298 & $(47,350)$ & 0.288 & $(136,305)$ & 0.520 \\
\hline & 3 & $(47,150,148)$ & 0.298 & $(152,350)$ & 0.291 & $(72,305)$ & 0.520 \\
\hline & 4 & $(48,150,41)$ & 0.338 & $(196,350)$ & 0.288 & $(192,305)$ & 0.520 \\
\hline & 5 & $(38,150,124)$ & 0.305 & $(221,350)$ & 0.288 & $(268,305)$ & 0.520 \\
\hline & Ave & - & 0.318 & - & 0.429 & - & 0.520 \\
\hline $\begin{array}{l}\text { Improved } \\
\text { algorithm }\end{array}$ & 1 & - & 0.351 & - & 0.709 & - & 0.520 \\
\hline
\end{tabular}

Note: The "-" means that matching objects cannot be found in the data set.

Table 3. Comparison of SC and F1 results of each algorithm on UCI data sets.

\begin{tabular}{ccccccc}
\hline \multirow{2}{*}{$\begin{array}{c}\text { UCI data } \\
\text { sets }\end{array}$} & \multicolumn{2}{c}{ K-means } & \multicolumn{2}{c}{ K-means++ } & \multicolumn{2}{c}{ Improved algorithm } \\
\cline { 2 - 7 } & SC & F1 & SC & F1 & SC & F1 \\
\hline Iris & 0.524 & 0.357 & 0.551 & 0.295 & 0.551 & 0.811 \\
Wine & 0.571 & 0.370 & 0.563 & 0.357 & 0.571 & 0.710 \\
Hayes-Roth & 0.571 & 0.346 & 0.571 & 0.354 & 0.572 & 0.436 \\
Ionosphere & 0.286 & 0.457 & 0.294 & 0.405 & 0.296 & 0.707 \\
Tae & 0.316 & 0.330 & 0.325 & 0.312 & 0.273 & 0.351 \\
Hagerman & 0.387 & 0.443 & 0.399 & 0.547 & 0.399 & 0.436 \\
\hline
\end{tabular}


Table 4. Clustering time comparison of all algorithms on UCI data sets.

\begin{tabular}{cccc}
\hline UCI data sets & K-means(ms) & K-means++(ms) & Improved algorithm(ms) \\
\hline Iris & 0.220 & 0.245 & 5.978 \\
Wine & 0.312 & 0.207 & 7.979 \\
Hayes-Roth & 0.193 & 0.360 & 6.017 \\
Ionosphere & 0.333 & 0.460 & 31.959 \\
Tae & 0.291 & 0.358 & 6.019 \\
Hagerman & 0.418 & 0.527 & 8.420 \\
\hline
\end{tabular}

\subsection{Results analysis}

It can be seen in Table 2, in the experimental data set in this paper, the clustering results improved stability obviously than the traditional K-means algorithm and $\mathrm{K}$-means++ algorithm. This is due to the calculation of the improved algorithm is based on the limit range and scope of the distance. That is to say, the result is certain, not volatile, so the stability of calculation is better than K-means algorithm and $\mathrm{K}$-means++. In terms of accuracy, the clustering result accuracy of the improved algorithm in this paper is better than that of the traditional K-means algorithm and K-means ++ average. Because the initial clustering center of the improved algorithm is unique and has certain anti-interference ability. Combined with the data information in Table 1, the algorithm is not sensitive to the difference of sample size and density. And it has high adaptability to the difference of sample size and density. The final clustering effect is also good. Compared with the traditional K-means algorithm, the contour coefficients of the algorithm clustering results in this paper are mostly greater than or equal to the contour coefficients of the traditional K-means algorithm and K-Means++ algorithm. And compared with the traditional K-means algorithm and K-Means++ algorithm, the improved algorithm has excellent F1 value.

The convergence rate of the improved algorithm mainly depends on the selection of the initial cluster centers and the number of clusters. For different data sets, the running time comparison of the algorithm is shown in Table 4. From clustering time contrast can be seen in Table 4, compared to the traditional K-means algorithm, the K-means++ algorithm, the improved algorithm requires more execution time. This is because the initial clustering center as the pretreatment.

The complexity analysis of the improved algorithm is mainly composed of two parts. The first part is the center point optimization process of the initial clustering, and then the clustering process is carried out. The total time complexity of the improved algorithm is $\mathrm{O}(\mathrm{N}(\mathrm{K}+\mathrm{N} / \mathrm{K}-2)--\mathrm{N} 2 / \mathrm{k} 2+\mathrm{K} \times \mathrm{m} \times \mathrm{d} \times \mathrm{n})$, which is longer than the time complexity of the traditional K-means algorithm. The space complexity is $\mathrm{O}(\mathrm{n} / \mathrm{K} \times \mathrm{m}+\mathrm{m} \times \mathrm{n})$, which is greater than that of the traditional K-means algorithm, where $\mathrm{N}$ is the number of data objects, $\mathrm{K}$ is the number of cluster classes, $\mathrm{M}$ is the number of fields of each data object, and $\mathrm{d}$ is the number of iterations.

Based on the above analysis, it can be seen that the algorithm presented in this paper has certain effects in processing contour data sets. The improved algorithm presented in this paper is reasonable, feasible and has unique clustering advantages, which can be used for cluster analysis of contour data.

But the improved algorithm time complexity is higher, algorithm running time is longer than the traditional K-means and K-means++algorithms. 


\section{Conclusion}

Traditional K-means clustering algorithm is widely used in data mining field. With the development of the times and the progress of technology, the arrival of the big data, just by the traditional algorithm is hard to meet the needs of a wide variety of data mining, including the type of a variety of data sets, and the need of algorithm performance, etc. In order to improve the algorithm of clustering effect; ensure the rationality of clustering; enhance the ability of processing contour shape data, this paper proposes a new optimization initial clustering center of the improved clustering algorithm. It provides an entirely new way to think about and process contour data. The farther apart the points are, the more likely they are to belong to different clusters and places with high density may have clustering centers. The experiment shows that the initial clustering center of the improved algorithm is stable. It avoids the problem of poor clustering effect caused by the sensitivity of the initial clustering center. And it has strong anti-outlier interference ability. It can effectively disperse the initial clustering center uniformly, and the improved algorithm has better clustering effect by comparing the value of the clustering evaluation index. Therefore, the improved algorithm is useful to process contour data, which provides a feasible, reasonable and effective method for the processing of discrete distributed data sets.

There are also some shortcomings for the proposed algorithm. Only several commonly used clustering data sets are analyzed, and there are still many places to study and think about the influence of the initial clustering center. The improved algorithm time complexity is higher, algorithm running time is longer, is not conducive to improving the efficiency of the algorithm. Moreover, more contour data are needed to verify potential problems and shortcomings of the algorithm. Based on this research, the next step is to improve the convergence and complexity of the algorithm, explore a better way to improve the efficiency of the algorithm, and improve the application of more kinds of data sets.

\section{References}

[1] Hai Mo. Overview of big data clustering algorithms [J]. Computer science, 2016, 43 (S1): 380-383.

[2] Wang Qian, Wang Cheng, FengZhenyuan, et al. Overview of K-means Clustering Algorithm Research [J]. Electronic design engineering, 2012, 20 (7): 21-24.

[3] Liu Wei. A Review of Clustering Algorithms in Data Mining [J]. Jiangsu Business Theory, 2018 (7) : 120-125.

[4] Ni Ping, Zhang Yuqing, Wen Guanxing, et al. Detection method of social botnet based on Group Characteristics [J]. Journal of the university of Chinese academy of sciences, 2014, 3 (5): 691-700.

[5] Ma Qian. Overview and Application of Clustering Algorithm [J]. China New Communications, 2012, 20 (14): 225-226.

[6] Xing Shuning, Liu Fangai, Zhao Xiaohui. An efficient parallel mining algorithm based on clustering [J]. Computer applications, 2016, 36(08): 2202-2206+2212.

[7] LuoJunfeng, Hong Dandan. A k-means clustering algorithm based on density and distance [J]. Software engineering, 2020, 23(10): 23-25+4.

[8] Li Qingxu, Chen Tianying, Hu Bo. New hierarchical clustering algorithm based on intersection [J]. Information technology and network security, 2020, 39(10): 18-22.

[9] Li Xiaoguang, Shao Chao. Density peak clustering algorithm based on grid data center [J]. Computer science, $2019,46(\mathrm{~S} 1): 457-460+487$.

[10] Wang Yidong. K-means algorithm optimization based on single point density [J]. Fujian computer, 2015, 31(01): 73-74+115

[11] Feng Jing, Mo Xiuliang, Wang Chundong. A Study of K-means Algorithm based on LDA in this essay clustering [J]. Journal of Tianjin University of Technology, 2008, 34(03): 7-11. 
[12] Yang Bo, Long Pengfei. Application of Condensed hierarchical clustering algorithm in intrusion detection [J]. Journal of Changsha Institute of Electric Power (Natural Science edition), 2005(03): 57-60.

[13] GuoYongkun, Zhang Xinyou, Liu Liping, Ding Liang, NiuXiaolu. K-means clustering Algorithm for Optimizing initial clustering center [J]. Computer Engineering and Applications, 2020, 56(15): $172-178$.

[14] DuanMingxiu, Tang Chaolin. Journal of jishou university (natural science edition), 2013, 34(01): 26-27.

[15] Mark Qin, Yang Yanjiao, Qin Hongwu, Geng Lin, Wang Pidong. K-means clustering algorithm combining maximum and minimum distance and weighted density [J]. Computer engineering and applications, 2020, 56(16): 50-54.

[16] Zhong Xi, Sun Xiang-e. Study on naive Bayesian integrated method based on K-means++ clustering [J]. Computer science, 2019, 46(S1): 439-441+451. 